SLAC-PUB-13792

\title{
Pandora's box and non-selfdual topological excitations
}

\author{
Mithat Ünsal \\ SLAC and Physics Department, Stanford University, Stanford, CA 94025/94305, USA
}

(Dated: March 23, 2010)

\begin{abstract}
In the last few years, we have realized the existence of a new class of topological excitations, which are rather distinct from the platonic world of monopoles, monopole-instantons and instantons. All of the latter arise as solutions of the Prasad-Sommerfield type first order differential (self-duality) equations and have been extensively discussed in the context of confinement and chiral symmetry breaking for the last 30 years. However, new calculable deformations of asymptotically free chiral and vector-like gauge theories give us a new picture of these physical phenomena. Most often, the excitations which lead to confinement are not solutions to PS-type equations, they are non-selfdual and they are often bizarre. They are referred to as magnetic bions, triplets, and quintets, due to their composite nature. Bizarre as they are, combined with large- $N$ volume independence, these novel non-self-dual excitations may also provide hope that at least some non-abelian gauge theories may be solvable.

Dedicated to Misha Shifman on the occasion of his sixtieth birthday. This write-up summarizes my interactions with him during our fruitful collaborations and important open problems in gauge theories.

PACS numbers:
\end{abstract}




\section{Contents}

I. Selfduality and non-selfduality: Tip and the iceberg? 2

A. Misha, smoothness conjecture and bions (almost) everywhere! 5

$\begin{array}{ll}\text { B. One cannot overstate the importance of the index theorem. } & 7\end{array}$

C. Chiral gauge theories and deformation program 9

D. La Belle Époque, the supersymmetric era and today 10

E. References and suggested reading 12

$\begin{array}{ll}\text { Acknowledgments } & 13\end{array}$

$\begin{array}{ll}\text { References } & 13\end{array}$

\section{The real voyage of discovery consists not in seeking new landscapes but in having new eyes. Marcel Proust.}

\section{SELFDUALITY AND NON-SELFDUALITY: TIP AND THE ICEBERG?}

A metaphor, even an imperfect one as described below, may often provide a useful insight into challenging situations. The story of Pandora's box (jar) is well-known. In its most common interpretation, Pandora opens the box due to curiosity and many evils, along with hope spread to earth. As this is an article not on moral but non-abelian gauge theories, the reader should replace the word evil with mystery or weirdness in what follows. (Although I must admit that gauge theories often have diabolical recipes for us.) Hope may remain the same, due to reasons which will become apparent.

I started to think about topological excitations in asymptotically free non-abelian gauge theories in early 2007. A distinguished member of such gauge theories, QCD, is perhaps one of the most challenging part of nature. The non-perturbative aspects of these theories have always been a source of fascination, excitement and doubled-curiosities. As described by Sasha Polyakov in his memoirs, perhaps mathematically the most important objects in addressing the non-perturbative aspects of gauge theories are instantons and monopoles. These topological excitations are solutions to first order self-duality equations, such as $F_{\mu \nu}=$ $\star F_{\mu \nu} \equiv \frac{1}{2} \epsilon_{\mu \nu \rho \sigma} F_{\rho \sigma}$ for the BPST instanton. Similarly, the monopoles or instanton-monopoles are solutions to the first order Prasad-Sommerfield type differential equations. When I 
started to think about topological excitations in gauge theories, my hunch was, there would in fact be more interesting and more important topological excitations pertinent to vectorlike and chiral gauge theories. The problem that we were unable to see these excitations was tied with the absence of controllable (and calculable) deformations and the right tools.

Around that time, I realized that such tools may indeed exist, if theories are compactified on a spatial (non-thermal) circle of size $L$. Due to the work of Gross, Pisarski and Yaffe from early 80's, I already knew that studying thermal compactifications would be beating a dead horse, so I did not care about it. My intuition was based on the sharp differences of quantum versus thermal fluctuations and I hoped that a more hospitable territory accessible via circle compactification existed.

With some optimism, I decided to examine $S U(2) \mathrm{QCD}(\operatorname{adj})$ (a decidedly low- $N$ theory) by taking advantage of a very unique and welcoming feature: its unbroken center symmetry ${ }^{1}$ even at arbitrarily small $S^{1} \times \mathbb{R}^{3}$. By the time I decided to do so, I did not know the index theorem for topological excitations on this geometry, but knew the famous APS-index (which applies on $\mathbb{R}^{4}$ ), and also knew lesser-known Callias (which applies on $\mathbb{R}^{3}$ ) index theorems. Combining the two, I was able to guess the form of the leading monopole operators. ${ }^{2}$ The results were rather surprising. Although monopoles were there and they were the leading topological excitations in the semi-classical expansion, they were not responsible for the mass gap for gauge fluctuations and confinement per se, which is counter to the mid-70's dream of confinement ̀̀ la Polyakov, 't Hooft, Mandelstam and others. I realized that I had stumbled upon a new class of topological excitations, but I did not first appreciate how general it could be. This realization was going to come from a phone call from Misha, to which I will return in the next section. First, I need to explain few aspects of these elusive topological excitations that revealed their existence in $\mathrm{QCD}(\operatorname{adj})$.

Let me briefly remind the reader the pair of relevant quantum numbers, useful in dis-

\footnotetext{
${ }^{1}$ The precursor of this idea was large $N$ volume independence of $\mathrm{QCD}(\operatorname{adj})$. A special form of volume independence is the famous Eguchi-Kawai reduction proposed in 1982. The first four dimensional working example appeared 25 years after the birth of the idea in 2007 and, as the reader may guess, it is $\mathrm{QCD}(\operatorname{adj})$ with periodic spin connection for fermions, a manifestly non-thermal compactification.

${ }^{2}$ As it should be clear, this was an educated guess. Later in 2008-CAQCD held in Minnesota, Erich Poppitz asked me a question about confinement in some gauge theories of his interest. My answer was, "if you tell me the index theorem for topological excitations, I will tell you what the answer is ..." I will return to this later.
} 
cussing topological excitations: magnetic charge and topological charge; $\left(\int F, \int F \widetilde{F}\right)$. On $\mathbb{R}^{3} \times S^{1}$, there are two types of monopoles, BPS and KK, as opposed to one (just BPS) as would be the case in the Polyakov model on $\mathbb{R}^{3}$. (This crucial aspect was realized in 1997 by Lee and Yi by using D-branes and Kraan and van Baal in gauge theory.) The existence of KK monopoles is due to compact topology of the holonomy. The charges of these excitations are BPS: $\left(1, \frac{1}{2}\right)$, KK: $\left(-1, \frac{1}{2}\right)$ with negations for the anti-monopoles: $\overline{\mathrm{BPS}}:\left(-1,-\frac{1}{2}\right)$, $\overline{\mathrm{KK}}:\left(1,-\frac{1}{2}\right)$. By using abelian duality, index theorem, and symmetries, I was able to show that the topological excitations which lead to confinement in this theory have charges $(2,0)$ and $(-2,0)$. Those familiar with topological excitations may find this strange for multiplereasons: (a)Unlike instantons or monopoles, these new objects have vanishing topological charge. In this sense, they are indistinguishable from perturbative vacuum! (b) They are not solutions to Prasad-Sommerfield type equations and hence they are manifestly non-self-dual.

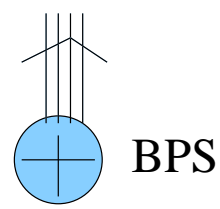

$(1,1 / 2)$
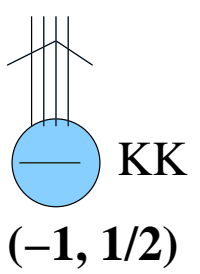

\section{Magnetic monopoles}

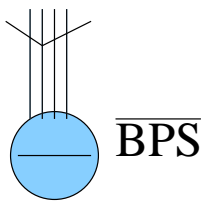

$(-1,-1 / 2)$

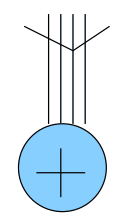

\section{$\overline{\mathrm{KK}}$} $(1,-1 / 2)$

\section{Magnetic bions}

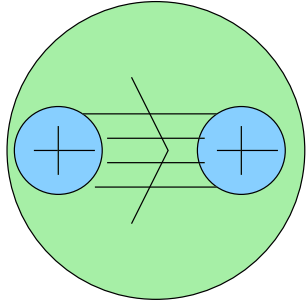

$(2,0)$

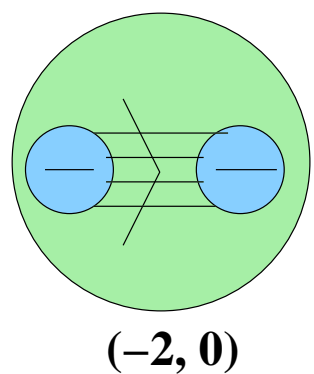

FIG. 1: Cartoon of the leading topological excitations in $S U(2) \mathrm{QCD}(\operatorname{adj})$ with $n_{f}=2$. The BPS and KK operators are $e^{i \sigma} \psi^{4}$ and $e^{-i \sigma} \psi^{4}$, where $\sigma$ is dual photon and $\psi$ is fermion zero mode. Flavor indices are suppressed. The product BPSKK is the usual instanton operator, $\psi^{8}$, with eight zero modes as per APS-index theorem. Because of the fermion zero modes, monopole operators cannot generate a mass gap for gauge fluctuations and confinement. Magnetic bions carry non-vanishing magnetic but zero topological charge. This corresponds to the $e^{2 i \sigma}+e^{-2 i \sigma}$ operator, which is the root cause of the mass gap for gauge fluctuations and confinement.

I referred to this topological excitation as magnetic bion as it is a composite, bound-state 
of BPS and $\overline{\mathrm{KK}}$ monopoles, shown in Fig.1. There is a puzzle here though: The constituents (each of which has charge +1 ) interact repulsively with a $1 / r$ potential induced by photon exchange and there are no other apparent force carriers in the system. Perhaps, the biggest surprise that I came to grips with in this theory was that the bions were stable due to a fermionic pairing mechanism, induced by fermion zero-mode exchange. This exchange generates a $2 n_{f} \log r$ attraction, which means a combined potential, $V_{\mathrm{BPS}-\overline{\mathrm{KK}}}(r)=\frac{1}{r}+2 n_{f} \log r$. This attraction implies the dynamical stability of magnetic bions. ${ }^{3}$ The long-distance dual theory made it manifest that the magnetic bions were the root cause of confinement and the mass gap in gauge sector of QCD(adj).

\section{A. Misha, smoothness conjecture and bions (almost) everywhere!}

The day after I posted the magnetic bion paper, I received an early morning call from Misha. Misha was pleased with the very simple fact that these excitations were there, but he was not surprised that their existence were not realized earlier. He tied the elusiveness of these objects to their indistinguishability from the perturbative vacuum in the sense of topological charge. Of course, no sensible gauge theorist with a sound mind would go out to search for topological excitations with $\int F \tilde{F}=0$ property! To my view, we were simply lucky that these non-self-dual excitations arose so naturally in QCD(adj). Misha has his very unique perspective and deep intuition on strong coupling gauge dynamics, and he was telling me something that I may have not appreciated/pursued otherwise: His main point was inspired from the large- $N$ (planar) orbifold/orientifold equivalences. The essence of his argument was following:

"The magnetic bions are surely there in the weak coupling - small $L$-domain;

presumably, they must also exist in the large $L$ domain, but in a non-dilute form.

At large $L$, there is an exact non-perturbative equivalence between $\mathrm{QCD}(\operatorname{adj})$

\footnotetext{
${ }^{3}$ Later, Larry Yaffe pointed out to me that similar fermion zero mode induced pairing $\left(\sim n_{f} \log r\right.$ attraction) of topological excitations, in an instanton-anti-instanton molecule, was found earlier by Callan, Dashen and Gross in the mid 70's. Apart from this similarity, there are no others between these composites. Bions, in particular, have a net magnetic charge and generate a mass gap in gauge sector. On the other hand, instanton-anti-instanton molecules are not of any particular importance for confinement.
} 
and $\mathrm{QCD}(\mathrm{AS} / \mathrm{S} / \mathrm{BF}) .{ }^{4}$ Thus, the magnetic bions must also exist in these theories.

How can we make them visible?"

Implicit to Misha's question was the staggering dissimilarity between QCD(adj) and $\mathrm{QCD}(\mathrm{AS} / \mathrm{S} / \mathrm{BF})$ in the small $L$ domain. All of the latter theories exhibited breaking of their center at small $L$ and were decidedly unlike QCD(adj). We needed to come-up with something which would disclose the semi-classical domain of these theories.

Incidentally, I was thinking on a proposal which makes large $N$ volume independence work all the way down to arbitrarily small $L>0$ for YM theory, in collaboration with Larry Yaffe. Motivated by adjoint fermion induced stabilization of center symmetry, we had the idea of using the double-trace deformations of YM theory to satisfy volume independence. This turns out to be a very interesting trick due to what I will call,

First magic of double-trace deformation: At large $N$, double-trace deformations of the action are an order $N^{2}$ effect and prevent center-breaking of YM theory on small $S^{1}$. On the other hand, the observables of YM theory (in the confined phase) and the ones of deformed YM theory for any $L>0$ differ only by $O\left(1 / N^{2}\right)$ effects in the large $N$ limit. In a sense, the deformation, after doing the good deed, sequesters itself. ${ }^{5}$

One should note that this is a very peculiar deformation, as typical $O\left(N^{2}\right)$ deformations of the action would alter the dynamics of the theory completely. Misha wanted to know about the small $N$ and small $L$ incarnations of such deformations. We thought for few days and had doubts about pursuing that direction: Our concern was the apparent non-locality of the Wilson line deformation. But we realized that this was an aesthetic issue. In fact, such deformed theories have nice renormalizable UV completions which yield our deformed action upon integrating out heavy KK modes in the small circle limit, and even without such, there is no harm to view these small $S^{1} \times \mathbb{R}^{3}$ theories as effective $3 \mathrm{~d}$ theories. This leads to the second magic of double-trace deformations.

Smoothness conjecture: At small or finite $N$, a deformation could be "engineered" in such a way that a sub-class of QCD-like theories on $\mathbb{R}^{4}$ could be smoothly connected to the deformed-QCD (QCD*) on arbitrarily small $S^{1} \times \mathbb{R}^{3}$.

\footnotetext{
4 These are YM theories with $n_{f}$ anti-symmetric, symmetric or bi-fundamental representation Dirac fermions. $\mathrm{QCD}(\mathrm{AS})$ is same as $\mathrm{QCD}(\mathrm{F})$ for $N=3$, thus a natural large $N$ limit thereof.

${ }^{5}$ The last sentence is coined by Gabriele Veneziano in a private conversation at GGI, Florence.
} 
Needless to say, this is what we were looking for despite the aesthetic issues. Regardless, this was a useful tool enabling us to move forward and identify interesting topological excitations. In the mean-time, we also learned about the existence of a very interesting lattice gauge theory work by Myers and Ogilvie. These authors have independently proposed deformations to study phases of partial center symmetry breaking and realized that, in a lattice formulation, the center symmetric phase can be continued to arbitrarily small $S^{1}$. Hence, with this non-perturbative confirmation, we decided to move on. This latter part is the main benefit of the "deformation program": It helps us to reveal the topological excitations in gauge dynamics.

Immediately, we were able to solve all interesting one-flavor QCD* theories on small $S^{1} \times \mathbb{R}^{3}$, and analytically demonstrate the existence of a mass gap, confinement and discrete chiral symmetry breaking. This was done by pin-pointing the non-perturbative source of each effect. Non-perturbative aspects which are quite hard in the original theory become straightforward tree-level phenomena upon a duality transform. Magnetic bions are ubiquitous, bearing responsibility for the mass gap, generally speaking, in almost all QCD-like theories with 2-index representation fermions.

\section{B. One cannot overstate the importance of the index theorem.}

Despite these solutions, neither Misha nor myself knew the exact index theorem for topological excitations on $S^{1} \times \mathbb{R}^{3}$, but we were able to proceed by using intuition based on the Callias and APS index theorems and, more often than not, bions were responsible for mass gap generation. The exact form of the index theorem is crucial to reveal the origin of confinement, which depends on the matter content of the theory! (This is again an unexpected aspect that we learned recently.)

There were a number of interesting gauge theories that Misha and I were unable to address, and this was due to the absence of a useful form of index theorem. I found a mathematics paper in the Differential Geometry arXiv by Nye and Singer, which addressed an index theorem for Dirac operator on $S^{1} \times \mathbb{R}^{3}$. Both Misha and I highly appreciated this paper and advertised this work in our paper. However, it was very hard for me to get a useful form of the index formula which we could use for concrete gauge theory applications. Eventually, Erich Poppitz and I (but mostly him) provided a new, insightful and elegant 


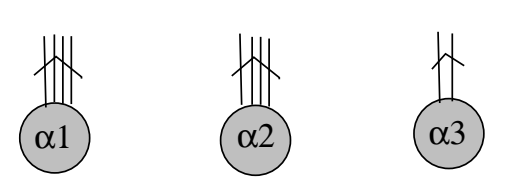

a) Magnetic monopoles

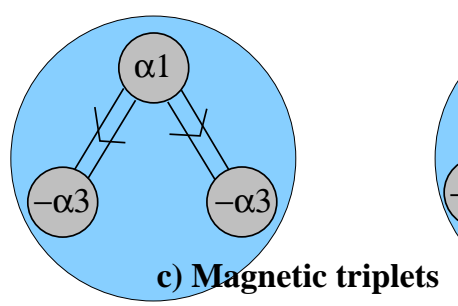

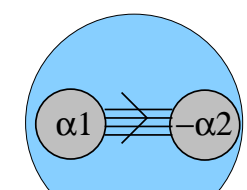

b) Magnetic bion

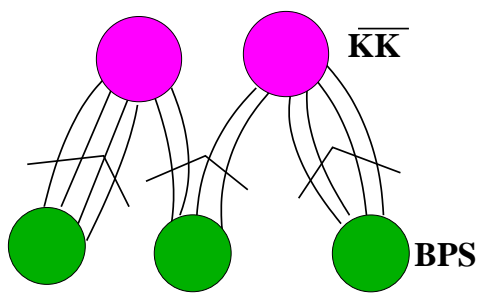

d)Magnetic quintet

FIG. 2: $S U(3)$ QCD(S): a)monopoles with indices $\left(I_{1}, I_{2}, I_{3}\right)=(4,4,2)$ do not induce a mass gap in the gauge sector because of their zero-modes. Note that $I_{\text {inst }}=\sum_{i=1}^{3} I_{i}=10$. b) magnetic bions and c) magnetic triplets generate a mass gap for gauge fluctuations. Note that all zero-modes need to be soaked up for the composite to contribute to a mass gap for gauge fluctuations. d) $S U(2)$ chiral theory with $I=3 / 2$ representation fermion and a cartoon of a magnetic quintet. $\left(I_{\mathrm{BPS}}, I_{\mathrm{KK}}\right)=(4,6)$. Note $I_{\text {inst. }}=\sum_{i=1}^{2} I_{i}=10$. The magnetic quintet is a composite of 3 BPS and $2 \overline{\mathrm{KK}}$ monopoles. In the absence of index theorem on $\mathbb{R}^{3} \times S^{1}$, it would perhaps be very hard, if not impossible, to guess these purely bosonic topological excitations, which generate confinement. The magnetic quintet is flipped from its original figure $\mathcal{W}$ to an $\mathcal{M}$ (isha) for the occasion.

derivation of the index formula, by using axial current non-conservation - an exact operator identity valid on any four-manifold - and a center-symmetric holonomy. We were in part being influenced by E. Weinberg's work on the Callias index theorem on $\mathbb{R}^{3}$. The derivation also provided a useful and simple form of the index formula which is easy to use for concrete (both supersymmetric and non-supersymmetric) gauge theory applications.

In order for the reader to appreciate how weird things can get regarding confinement, and why the index formula is so important, consider two gauge theories: Vector-like $S U(3)$ $\mathrm{QCD}(\mathrm{S})$ and chiral $S U(2)$ with an $I=3 / 2$ Weyl fermion. I picked these two examples, because both theories have $I_{\text {inst. }}=10$ zero modes in the background of an instanton. The topological excitations, zero mode structures and the non-selfdual excitations which generate the mass gaps are shown in Fig. 2. This figure is the reason why knowing the index theorem is so important in our understanding of gauge theories. The actions of various topological 
excitations, in a center-symmetric background, relative to an instanton are given by:

$$
\left\{S_{\text {mon }}, S_{\text {bion }}, S_{\text {triplet }}, S_{\text {quintet }}\right\}=\left\{\frac{1}{N}, \frac{2}{N}, \frac{3}{N}, \frac{5}{N}\right\} \times\left(S_{\text {inst }}=\frac{8 \pi^{2}}{g^{2}}\right) .
$$

Moreover, since $e^{-S_{\text {inst }}} \sim e^{-N}$ and $e^{-S_{\text {mon } / \text { bion }}} \sim e^{-1}$ in $N$-counting, the leading (composite) topological excitations, unlike instantons, are not suppressed in the large- $N$ limit.

\section{Chiral gauge theories and deformation program}

Around this time, I had another phone conversation with Misha. I was thinking of pursuing the deformation program for multi-flavor QCD-like theories due to an exotic phenomena I had in mind: I wanted to show that QCD-like theories may generically possess phases of confinement without chiral symmetry breaking on $S^{1} \times \mathbb{R}^{3}$, a phenomena first realized in QCD(adj). But this time, Misha had something different in his mind: Chiral gauge theories. On the phone, I remember him saying that "this is an uncharted territory" despite chiral theories being "familiar" for almost three decades. Lattice does not work, string theory is not useful so far and large $N$ techniques are not useful either. The only known thing is the 't Hooft anomaly matching, while being one of the most powerful results in gauge theories, is not a dynamical framework. (In fact, there are examples of misleading anomaly matchings, that is to say, despite the matching of UV anomalies by some IR "composites", there are cases in which such composites do not describe the IR-physics.)

In a short time, Misha and I studied two main classes of chiral gauge theories by using our deformation program: Type I, chiral quiver theories and Type II, $S U(N)$ chiral theory with one AS , and $N-4$ anti-fundamental representation left-handed Weyl fermions. Currently, deformation theory is the only microscopic framework to understand the dynamics of these gauge theories.

Because of center-symmetric holonomy and abelianization, one expects topologically stable monopoles. We discovered a very strange phenomena (which never occurs in QCD-like theories). The monopole operator contributions to the non-perturbative dynamics of chiral gauge theories vanish identically! ${ }^{6}$ Upon averaging over all zero modes, in particular the

\footnotetext{
${ }^{6}$ In particular, for type II theories, monopole operators are almost all fermionic and the index is in general odd(!), similar to the number of instanton zero modes in an $S U(2)$ gauge theory with an odd-number of doublets. The latter class of theories, as shown by Witten, have a global anomaly and do not exist as
} 
global $U(1)$ angles, all monopole operators drop out. On the other hand, one can show that new topological excitations with more involved fermion zero-mode structure (monopole-ring operators) and bions are still there. They govern, respectively, chiral symmetry realization and mass gap for gauge fluctuations.

\section{La Belle Époque, the supersymmetric era and today}

I think that our understanding of non-perturbative aspect of non-supersymmetric gauge theories has seen a drastic change in the last two years in an unanticipated way. It is unanticipated because earlier solutions/scenarios of confinement generally relied on selfdual topological excitations. Our solutions for vector-like and chiral theories indicate that it is often non-self-dual topological excitations which generate a mass gap in the gauge sector and confinement.

To put things into context, I would like to tell my opinions regarding past works on confinement in non-abelian gauge theories. Of course, la belle époque of this problem is the mid-70s to early 80s. To me, the most important solution of that era is the one of Polyakov, where he mapped the partition function of a gauge theory to a dilute gas of self-dual monopoles (3d -instantons). The dual superconductivity scenario of 't Hooft and Mandelstam was also born during that era, but had to wait almost two decades for an explicit construction, eventually being realized in the very elegant solution of Seiberg-Witten, in '94, in the supersymmetric context. In both cases, the self-dual (BPS) objects play crucial roles.

When I realized the existence of magnetic bions, I did not think that they could be this generic in gauge theories. Misha's intuition, the use of double-trace deformations and the relevant index theorem changed my opinion. Now, I think that the magnetic bions and similar non-selfdual topological excitations are very likely the most important topological configurations in typical gauge theories. These objects are rather messy, mysterious and often bizarre, as shown in Fig.1 and Fig.2. ${ }^{7}$ But now, we have the correct mathematical tools to study them rather precisely, on $\mathbb{R}^{3} \times S^{1}$, where the theory is locally four dimensional.

consistent quantum theories. In our case, there are no such global anomalies and these are fine quantum theories.

7 It would be interesting to see whether the non-self-dual topological excitations such as magnetic bions, triplets, etc. have a counter-part in string theory, and whether they could be useful there as well. 
The world of these new non-selfdual topological excitations is almost contrary to the platonic world of self-dual monopoles, instantons, and the dreams of the past. Returning to the title of this work, I think the Pandora's box for QCD and chiral theories is now open, or at least ajar, for the first time, so that we can have a glimpse of gauge theories' inner goings-on. Many interesting things just happen to be in there and I am sure we will see more. I also think that the combination of these novel topological excitations and another unexploited concept from the past, the large- $N$ volume independence, carry hope that we can come to a fuller understanding of most of these gauge theories.

This is, of course, an idiosyncratic point of view. Many people today hope that, gauge/string duality may provide useful insights to gauge dynamics in four dimensions. Perhaps important problems like conformality, confinement, and the mass gap in YM theories with or without fermionic matter fields will be understood by geometrization. Some prominent string theorists even argue that this is the best analytic approach at hand now, but I disagree with these claims for good reasons. When I started to think about topological excitations in gauge theories, the reason I did not pursue a stringy approach was due to my belief that one could construct new microscopic field theory techniques which could be more beneficial than what string theory can do under similar circumstances. ${ }^{8}$ I think that a few people, including Misha and me, partially achieved this goal by revealing the existence of new non-selfdual topological excitations and their role in confinement.

\footnotetext{
${ }^{8}$ Polyakov mentions in his scientific memoirs that the reason he decided to move from semi-classical techniques to the string description was the belief that the semi-classical approach cannot fully describe the strong coupling regime of QCD. I think this is most likely true. But the caveat here is that all known string theory descriptions of QCD-like theories either have non-decoupling states which prevent them from reducing to pure large- $N$ QCD, or they are phenomenological (bottom-up) in their nature. The latter approach is not useful for the type of questions that I find interesting in gauge theories. The first may be fine modulo its caveats. In my view, the importance of semi-classical techniques is no less than any string theory approach, perhaps more. They are microscopic, can address finite $N$, and have taught us about the existence of new mechanisms of confinement, conformality, non-self-dual topological excitations and new scales in QCD-like theories. This is a sufficiently good reason to argue that this approach merits more attention.
} 


\section{E. References and suggested reading}

Since this short note is written as a scientific memoir of my interactions with Misha and describes our program based on recent papers, I did not attempt to give references. Instead, I will mention a few papers which influenced my thinking about gauge theories on $\mathbb{R}^{3} \times S^{1}$, and a few references that may be useful to guide interested readers.

Ref [1] : This is the first use of duality in a relativistic gauge theory, and establishes the role of monopoles in confinement.

Ref [2] : Polyakov model with adjoint fermions; and a must-read complement of Ref [1] . The authors relies more on symmetries than dynamical aspects. Consequently, physical interpretation of some topological excitations are missing. These are explained in Ref [6].

Ref [3] : A beautiful field theory discussion of $\mathcal{N}=1 \mathrm{SYM}$ on $S^{1} \times \mathbb{R}^{3}$, in the sprit of the first two, and one of the papers that I like most. The gaps regarding the interpretation of confinement in $\mathcal{N}=1 \mathrm{SYM}$ are filled in Ref [6] .

Ref $[4,5]$ : Introduced KK-monopole, a self-dual monopole-instanton, and an essential ingredient of the magnetic bion mechanism.

Ref $[6]$ : This is the first analytic solution of a locally four dimensional non-abelian (non-supersymmetric) gauge theory on $S^{1} \times \mathbb{R}^{3}$. It introduced magnetic bions, a new class of non-self-dual topological excitations. Its discussion about $\mathcal{N}=1 \mathrm{SYM}$ should be viewed as a complement to Ref [3].

Ref $[7]$ : This index theorem is the most crucial result that will shape the understanding of gauge theories on $S^{1} \times \mathbb{R}^{3}$. However, it is hard to read and the way the results are expressed are not particularly useful for gauge theory applications. The index theorem here is far more refined than the familiar Atiyah-Singer index theorem for instantons on $\mathbb{R}^{4}$. This paper is so far not appreciated in the physics literature.

Ref $[8]$ : Gives a new derivation of the index theorem by using an exact operator identity - axial-current non-conservation - valid on any four-manifold, and by paralleling E. Weinberg's discussion of the Callias index on $\mathbb{R}^{3}$. It also provides a calculation of the index in specific backgrounds and a discussion of its jumps, properties which are of interest for concrete gauge theory application.

Ref $[9,10]$ : The first paper is where double-trace deformations are introduced to formulate the smoothness conjecture. This construction allowed us to see that magnetic bions 
were pertinent to most interesting gauge theories, chiral or vector-like. The use of the deformations and the index theorem essentially puts the understanding provided in non-susy QCD or even chiral theories more or less on the same level with SQCD of Ref [13].

Ref [11] : Deformed YM theory can be used to study phases of partial center symmetry breaking on lattice, and a sufficiently large deformation stabilizes the center symmetry at any value of the bare lattice coupling. See the beautiful Fig.1 therein.

Ref $[12]$ : Fully center-stabilized deformations of large- $N$ YM theories obey volume independence.

Ref [13] : For SQCD, the smoothness conjecture is exact. The vacuum of the theory on $S^{1} \times \mathbb{R}^{3}$ knows about the vacuum structure on $\mathbb{R}^{4}$. This paper has many good examples of the derivation of non-perturbative superpotentials by using holomorphy and symmetry. Using the index theorem provides a microscopic derivation of some of these superpotentials.

\section{Acknowledgments}

This work was supported by the U.S. Department of Energy Grant DE-AC02-76SF00515.

[1] A. M. Polyakov, "Quark Confinement And Topology Of Gauge Groups," Nucl. Phys. B 120, 429 (1977).

[2] I. Affleck, J. A. Harvey and E. Witten, "Instantons And (Super)Symmetry Breaking In (2+1)-Dimensions," Nucl. Phys. B 206, 413 (1982).

[3] N. M. Davies, T. J. Hollowood, V. V. Khoze and M. P. Mattis, “ Gluino condensate and magnetic monopoles in supersymmetric gluodynamics," Nucl. Phys. B 559, 123 (1999) [arXiv:hepth/9905015].

[4] K. M. Lee and P. Yi, " Monopoles and instantons on partially compactified D-branes," Phys. Rev. D 56, 3711 (1997) [arXiv:hep-th/9702107].

[5] T. C. Kraan and P. van Baal, Nucl. Phys. B 533, 627 (1998) [arXiv:hep-th/9805168].

[6] M. Ünsal, "Magnetic bion condensation: A new mechanism of confinement and mass gap in four dimensions," Phys. Rev. D 80, 065001 (2009) [arXiv:0709.3269 [hep-th]]. 
[7] T. M. W. Nye and M. A. Singer, "An $L^{2}$-Index Theorem for Dirac Operators on $S^{1} \times R^{3}$," arXiv:math/0009144.

[8] E. Poppitz and M. Ünsal, "Index theorem for topological excitations on $R^{3} * S^{1}$ and ChernSimons theory," JHEP 0903, 027 (2009) [arXiv:0812.2085 [hep-th]].

[9] M. Shifman and M. Ünsal, "QCD-like Theories on $R_{3} \times S_{1}$ : a Smooth Journey from Small to Large $r\left(S_{1}\right)$ with Double-Trace Deformations," Phys. Rev. D 78, 065004 (2008) [arXiv:0802.1232 [hep-th]].

[10] M. Shifman and M. Ünsal, "On Yang-Mills Theories with Chiral Matter at Strong Coupling," Phys. Rev. D 79, 105010 (2009) [arXiv:0808.2485 [hep-th]].

[11] J. C. Myers and M. C. Ogilvie, "New Phases of SU(3) and SU(4) at Finite Temperature," Phys. Rev. D 77, 125030 (2008) [arXiv:0707.1869 [hep-lat]].

[12] M. Ünsal and L. G. Yaffe, "Center-stabilized Yang-Mills theory: confinement and large $N$ volume independence," Phys. Rev. D 78, 065035 (2008) [arXiv:0803.0344 [hep-th]].

[13] O. Aharony, A. Hanany, K. A. Intriligator, N. Seiberg and M. J. Strassler, "Aspects of N = 2 supersymmetric gauge theories in three dimensions," Nucl. Phys. B 499, 67 (1997) [arXiv:hepth/9703110]. 